\title{
Chaplygin gas dominated anisotropic brane world cosmological models
}

\author{
M. K. Mak* and T. Harkd \\ Department of Physics, The University of Hong Kong, Pok Fu Lam Road, Hong Kong
}

(Dated: June 24, 2018)

\begin{abstract}
We present exact solutions of the gravitational field equations in the generalized Randall-Sundrum model for an anisotropic brane with Bianchi type I geometry, with a generalized Chaplygin gas as matter source. The generalized Chaplygin gas, which interpolates between a high density relativistic era and a non-relativistic matter phase, is a popular dark energy candidate. For a Bianchi type I space-time brane filled with a cosmological fluid obeying the generalized Chaplygin equation of state the general solution of the gravitational field equations can be expressed in an exact parametric form, with the comoving volume taken as parameter. In the limiting cases of a stiff cosmological fluid, with pressure equal to the energy density, and for a pressureless fluid, the solution of the field equations can be expressed in an exact analytical form. The evolution of the scalar field associated to the Chaplygin fluid is also considered and the corresponding potential is obtained. The behavior of the observationally important parameters like shear, anisotropy and deceleration parameter is considered in detail.
\end{abstract}

PACS numbers: 04.50.+h, 04.20.Jb, 04.20.Cv, 95.35.+d

\section{INTRODUCTION}

The observations of high redshift supernovae 1] and the Boomerang/Maxima data 2], showing that the location of the first acoustic peak in the power spectrum of the microwave background radiation is consistent with the inflationary prediction $\Omega=1$, have provided compelling evidence for a net equation of state of the cosmic fluid lying in the range $-1 \leq w=p / \rho<-1 / 3$. To explain these observations, two dark components are invoked: the pressureless cold dark matter $(\mathrm{CDM})$ and the dark energy (DE) with negative pressure. CDM contributes $\Omega_{m} \sim 0.3$,and is mainly motivated by the theoretical interpretation of the galactic rotation curves and large scale structure formation. DE is assumed to provide $\Omega_{D E} \sim 0.7$ and is responsible for the acceleration of the distant type Ia supernovae. There are a huge number of candidates for DE in the literature (for recent reviews see [3] and [4]). However, neither CDM nor DE has direct laboratory observational or experimental evidence for its existence. It would be important if a unified dark matter -dark energy scenario could be found, in which these two components are different manifestations of a single fluid [5].

A candidate for such an unification is the so-called generalized Chaplygin gas, which is an exotic fluid with the equation of state $p=-B / \rho^{\alpha}$, where $B$ and $\alpha$ are two parameters to be determined. It was initially suggested by Kamenshchik, Moschella and Pasquier [6] with $\alpha=1$, and then generalized by Bento, Bertolami and Sen [7] for the case $\alpha \neq 1$. The Chaplygin gas also appears in the stabilization of branes in Schwarzschild-AdS black hole bulks as a critical theory at the horizon [8] and in the stringy analysis of black holes in three dimensions [9]. The Chaplygin equation of state can also be derived from Born-Infeld type Lagrangians [7], 11]. This simple and elegant model smoothly interpolates between a non-relativistic matter phase $(p=0)$ and a negative-pressure dark energy dominated phase.

The cosmological implications of the Chaplygin gas model have been intensively investigated in the recent literature [12].

The Chaplygin gas cosmological model has been constrained by using different cosmological observations, like type Ia supernovae [13], 14], the CMB anisotropy measurements [15], gravitational lensing surveys [16], the age measurement of high redshift objects [17] and the X-ray gas mass fraction of clusters [18]. The obtained results are somewhat controversial, with some of them claiming good agreement between the data and the Chaplygin gas model, while the rest ruling it as a feasible candidate for dark matter. In particular, the standard Chaplygin gas model with $\alpha=1$ is ruled out by the data at a $99 \%$ level [18].

The embedding approach to gravity, according to which our four-dimensional space-time is a three-brane, embedded in a five-dimensional space-time (the bulk), has a long history, beginning at least with the classic book by Eisenhart [19]. More recently, this idea was considered by Akama 20] and Rubakov and Shaposhnikov [21] (an exhaustive list of references on the early articles published in this field can be found in Bandos and Kummer [22] and Pavsic and

*Electronic address: mkmak@vtc.edu.hk

†Electronic address: harko@hkucc.hku.hk 
Tapia [23], respectively). Recently, due to the proposal by Randall and Sundrum 24] the idea of the embedding of our Universe in a higher dimensional space had attracted again a considerable interest. According to the brane-world scenario, the physical fields (electromagnetic, Yang-Mills etc.) in our four-dimensional Universe are confined to the three brane. These fields are assumed to arise as fluctuations of branes in string theories. Only gravity can freely propagate in both the brane and bulk space-times, with the gravitational self-couplings not significantly modified. This model originated from the study of a single 3-brane embedded in five dimensions, with the $5 D$ metric given by $d s^{2}=e^{-f(y)} \eta_{\mu \nu} d x^{\mu} d x^{\nu}+d y^{2}$, which, due to the appearance of the warp factor, could produce a large hierarchy between the scale of particle physics and gravity. Even if the fifth dimension is uncompactified, standard $4 D$ gravity is reproduced on the brane. Hence this model allows the presence of large, or even infinite non-compact extra dimensions. Our brane is identified to a domain wall in a 5-dimensional anti-de Sitter space-time. For a review of dynamics and geometry of brane Universes see [25].

Due to the correction terms coming from the extra dimensions, significant deviations from the Einstein theory occur in brane world models at very high energies [26]. Gravity is largely modified at the electro-weak scale $1 \mathrm{TeV}$. The cosmological implications of the brane world theories have been extensively investigated in the physical literature [27]. Gravitational collapse can also produce high energies, with the five dimensional effects playing an important role in the formation of black holes [28].

The possibility that the non-local effects, generated by the free gravitational field of the bulk in a conformally symmetric brane, may provide an explanation for the dynamics of the neutral hydrogen clouds at large distances from the galactic center, and hence provide a purely gravitational explanation for the existence of dark matter, was suggested in [29].

The behavior and the properties of the anisotropic Bianchi type I brane-world for different type of matter sources, including inflationary scalar fields, have been intensively investigated in the framework of the brane world cosmologies 30]. The study of anisotropic homogeneous brane world cosmological models have shown an important difference between these models and standard four-dimensional general relativity, namely, that brane Universes are born in an isotropic state. For Bianchi type I and V geometries this type of behavior has been found both by exactly solving the gravitational field equations [31], or from the qualitative analysis of the model [32]. A general analysis of the anisotropy in spatially homogeneous brane world cosmological models has been performed by Coley [33], who has shown that the initial singularity is isotropic, and hence the initial conditions problem is naturally solved. Consequently, close to the initial singularity, these models do not exhibit Mixmaster or chaotic-like behavior [34]. Based on the results of the study of homogeneous anisotropic cosmological models Coley conjectured that the isotropic singularity could be a general feature of brane cosmology. The analytic study of the values of the anisotropy in the limit of small times shows that for all Bianchi type space-times filled with a non-zero pressure cosmological fluid, obeying a barotropic equation of state, the initial singular state on the brane is isotropic 35]. This result is obtained by assuming that in the limit of small times the asymptotic behavior of the scale factors is of Kasner-type. For brane worlds filled with dust or with a scalar field, the initial value of the anisotropy coincides in both brane world and standard four-dimensional general relativistic cosmologies.

It is the purpose of this paper to investigate some classes of exact solutions of the gravitational field equations in the brane world model for the anisotropic Bianchi type I geometry, by assuming that the matter source on the brane consist of a generalized Chaplygin gas. In this case the general solution of the field equations can be obtained in an exact parametric form, for arbitrary values of the parameters characterizing the equation of state of the matter. The inclusion of the quadratic terms in the energy-momentum tensor of the perfect cosmological fluids leads to major changes in the early dynamics of the anisotropic Universe, as compared to the standard general relativistic case. We also consider the brane dynamics of the scalar field associated to the Chaplygin case and obtain the corresponding self-interaction potential. The behavior of the observationally important physical quantities like anisotropy, shear and deceleration parameters is considered in detail for all these models.

The present paper is organized as follows. The gravitational field equations for a Chaplygin gas on a brane with an anisotropic Bianchi type I geometry are written down in Section II. In Section III we present the general solution of the field equations. In Section III we discuss and conclude our results.

\section{GEOMETRY, MATTER AND FIELD EQUATIONS ON THE BRANE}

In the $5 D$ space-time the brane-world is located as $Y\left(X^{I}\right)=0$, where $X^{I}, I=0,1,2,3,4$ are 5 -dimensional coordinates. The effective action in five dimensions is [36]

$$
S=\int d^{5} X \sqrt{-g_{5}}\left(\frac{1}{2 k_{5}^{2}} R_{5}-\Lambda_{5}\right)+\int_{Y=0} d^{4} x \sqrt{-g}\left(\frac{1}{k_{5}^{2}} K^{ \pm}-\lambda+L^{\text {matter }}\right),
$$


where $k_{5}^{2}=8 \pi G_{5}$ is the 5 -dimensional gravitational coupling constant, $\Lambda_{5}$ is the vacuum energy in the bulk, $\lambda$ is the vacuum energy on the brane and where $x^{\mu}, \mu=0,1,2,3$ are the induced 4-dimensional brane world coordinates. $R_{5}$ is the $5 D$ intrinsic curvature in the bulk and $K^{ \pm}$is the intrinsic curvature on either side of the brane.

On the 5 -dimensional space-time (the bulk), with the negative vacuum energy $\Lambda_{5}$ and brane energy-momentum as source of the gravitational field, the Einstein field equations are given by

$$
G_{I J}=k_{5}^{2} T_{I J}, \quad T_{I J}=-\Lambda_{5} g_{I J}+\delta(Y)\left[-\lambda g_{I J}+T_{I J}^{\text {matter }}\right],
$$

In this space-time a brane is a fixed point of the $Z_{2}$ symmetry. In the following capital Latin indices run in the range $0, \ldots, 4$ while Greek indices take the values $0, \ldots, 3$.

Assuming a metric of the form $d s^{2}=\left(n_{I} n_{J}+g_{I J}\right) d x^{I} d x^{J}$, with $n_{I} d x^{I}=d \chi$ the unit normal to the $\chi=$ const. hypersurfaces and $g_{I J}$ the induced metric on $\chi=$ const. hypersurfaces, the effective four-dimensional gravitational equations on the brane (which are the consequence of the Gauss-Codazzi equations) take the form [26]:

$$
G_{\mu \nu}=-\Lambda g_{\mu \nu}+k_{4}^{2} T_{\mu \nu}+k_{5}^{4} S_{\mu \nu}-E_{\mu \nu}
$$

where $S_{\mu \nu}$ is the local quadratic energy-momentum correction

$$
S_{\mu \nu}=\frac{1}{12} T T_{\mu \nu}-\frac{1}{4} T_{\mu}^{\alpha} T_{\nu \alpha}+\frac{1}{24} g_{\mu \nu}\left(3 T^{\alpha \beta} T_{\alpha \beta}-T^{2}\right)
$$

and $E_{\mu \nu}$ is the nonlocal effect from the bulk free gravitational filed, transmitted projection of the bulk Weyl tensor $C_{I A J B}$

$$
E_{I J}=C_{I A J B} n^{A} n^{B}, \quad E_{I J} \rightarrow E_{\mu \nu} \delta_{I}^{\mu} \delta_{J}^{\nu} \quad \text { as } \quad \chi \rightarrow 0
$$

The four-dimensional cosmological constant, $\Lambda$, and the coupling constant, $k_{4}$, are given by

$$
\Lambda=\frac{k_{5}^{2}}{2}\left(\Lambda_{5}+\frac{k_{5}^{2} \lambda^{2}}{6}\right), \quad k_{4}^{2}=\frac{k_{5}^{4} \lambda}{6} .
$$

The Einstein equation in the bulk, Codazzi equation, also implies the conservation of the energy momentum tensor of the matter on the brane,

$$
D_{\nu} T_{\mu}^{\nu}=0
$$

Moreover, the contracted Bianchi identities on the brane imply that the projected Weyl tensor should obey the constraint

$$
D_{\nu} E_{\mu}{ }^{\nu}=k_{5}^{4} D_{\nu} S_{\mu}{ }^{\nu}
$$

Finally, the equations (3), (7) and (8) give the complete set field equations for the brane gravitational field.

For any matter fields (scalar field, perfect fluids, kinetic gases, dissipative fluids etc.) the general form of the brane energy-momentum tensor can be covariantly given as [25]

$$
T_{\mu \nu}=\rho u_{\mu} u_{\nu}+p h_{\mu \nu}+\pi_{\mu \nu}+2 q_{(\mu} u_{\nu)} .
$$

The decomposition is irreducible for any chosen 4 -velocity $u^{\mu}$. Here $\rho$ and $p$ are the energy density and isotropic pressure, and $h_{\mu \nu}=g_{\mu \nu}+u_{\mu} u_{\nu}$ projects orthogonal to $u^{\mu}$.

The energy flux obeys the relation $q_{\mu}=q_{<\mu>}$, and the anisotropic stress obeys $\pi_{\mu \nu}=\pi_{<\mu \nu>}$, where angular brackets denote the projected, symmetric and trace-free part:

$$
V_{<\mu>}=h_{\mu}{ }^{\nu} V_{\nu}, \quad W_{<\mu \nu>}=\left[h_{(\mu}{ }^{\alpha} h_{\nu)}{ }^{\beta}-\frac{1}{3} h^{\alpha \beta} h_{\mu \nu}\right] W_{\alpha \beta} .
$$

The symmetry properties of $E_{\mu \nu}$ imply that in general we can decompose it irreducibly with respect to a chosen 4-velocity field $u^{\mu}$ as 25]

$$
E_{\mu \nu}=-k^{4}\left[\mathcal{U}\left(u_{\mu} u_{\nu}+\frac{1}{3} h_{\mu \nu}\right)+\mathcal{P}_{\mu \nu}+2 \mathcal{Q}_{(\mu} u_{\nu)}\right]
$$


where $k=k_{5} / k_{4}, \mathcal{U}$ is a scalar, $\mathcal{Q}_{\mu}$ a spatial vector and $\mathcal{P}_{\mu \nu}$ a spatial, symmetric and trace-free tensor. For homogeneous models $\mathcal{Q}_{\mu}=0$ and $\mathcal{P}_{\mu \nu}=0$ [32]. Hence the only non-zero contribution from the 5-dimensional Weyl tensor from the bulk is given by the scalar or "dark radiation" term $\mathcal{U}$.

We consider that in Eq. (9) the heat transfer is zero, $q_{\mu}=0$ and we also neglect the effect of the possible anisotropic stresses (viscosity of the cosmological fluid, magnetic fields etc.) on the dynamics of the Universe. This allows to assume that the cosmological fluid on the brane is perfect, without dissipative effects. Then the matter correction terms are given by

$$
S_{\mu \nu}=\frac{1}{12} \rho^{2} u_{\mu} u_{\nu}+\frac{1}{12} \rho(\rho+2 p) h_{\mu \nu} .
$$

The line element of a Bianchi type I space-time, which generalizes the flat Friedmann-Robertson-Walker metric to the anisotropic case, is given by

$$
d s^{2}=-d t^{2}+a_{1}^{2}(t) d x^{2}+a_{2}^{2}(t) d y^{2}+a_{3}^{2}(t) d z^{2}
$$

We define the following variable:

$$
\begin{gathered}
V=\prod_{i=1}^{3} a_{i}, \\
H=\frac{1}{3} \sum_{i=1}^{3} H_{i}, \\
H_{i}=\frac{\dot{a}_{i}}{a_{i}}, i=1,2,3, \\
\Delta H_{i}=H_{i}-H, i=1,2,3 .
\end{gathered}
$$

In equations (14)-(17) $V$ is the volume scale factor, $H_{i}, i=1,2,3$ are the directional Hubble parameters and $H$ is the mean Hubble parameter. From Eqs. (14) and (16) we obtain the important relation $H=\dot{V} / 3 V$.

The physical quantities of observational interest in cosmology are the expansion scalar $\theta$, the mean anisotropy parameter $A$ and the shear scalar $\sigma^{2}$, and are defined according to

$$
\begin{gathered}
\theta=3 H \\
A=\frac{1}{3} \sum_{i=1}^{3}\left(\frac{\Delta H_{i}}{H}\right)^{2}, \\
\sigma^{2}=\frac{1}{2} \sigma_{i k} \sigma^{i k}=\frac{1}{2} \sum_{i=1}^{3} H_{i}^{2}-3 H^{2}=\frac{3}{2} A H^{2} .
\end{gathered}
$$

An important observational quantity is the deceleration parameter

$$
q=\frac{d}{d t}\left(\frac{1}{H}\right)-1
$$

The sign of the deceleration parameter indicates whether the model inflates or not. The positive sign of $q$ corresponds to "standard" decelerating models whereas the negative sign indicates inflation.

We assume that the isotropic pressure $p$ of the cosmological fluid on the brane obeys a modified Chaplygin gas equation of state [37],

$$
p=\gamma \rho-\frac{B}{\rho^{\alpha}}
$$


where $0 \leq \gamma \leq 1$ and $0 \leq \alpha \leq 1$. $B$ is a positive constant.

When $\gamma=1 / 3$ and the comoving volume of the Universe is small $(\rho \rightarrow \infty)$, this equation of state corresponds to a radiation dominated era. The main reason for the use of the modified Chaplygin gas equation of state is that it can also describe the very high density $(\gamma=1)$ and the radiation dominated phase in the evolution of the Universe. When the density is small, $\rho \rightarrow 0$, the equation of state corresponds to a cosmological fluid with negative pressure (the dark energy). In this limit, the modified Chaplygin equation of state corresponds to a mixture of cold dark matter and dark energy [7]. At all stages it shows a mixture of dark energy, dark matter and radiation. For $\rho=(B / \gamma)^{1 /(\alpha+1)}$ the matter content is pure dust with $p=0$. In the following we neglect the effect of baryonic (luminous) matter on the dynamics of the Universe, which, in a rigorous approach, should be added separately to the equation of state of the cosmological matter. But since the baryonic matter represents only $4-5 \%$ from the total amount of energy-matter in the Universe [3], [4], its effect on the large scale cosmological dynamics can be ignored.

The speed of sound $v_{s}^{2}=\partial p / \partial \rho$ in the Chaplygin gas is given by

$$
v_{s}^{2}=\gamma(1+\alpha)-\frac{\alpha p}{\rho} .
$$

Using the variables (14)-(17), the Einstein gravitational field equations, the Bianchi identity and the evolution equation for the nonlocal dark radiation take the form, respectively,

$$
\begin{aligned}
3 \dot{H}+\sum_{i=1}^{3} H_{i}^{2} & =\Lambda-\frac{k_{4}^{2}}{2}\left[(3 \gamma+1) \rho-\frac{3 B}{\rho^{\alpha}}\right]-\frac{k_{4}^{2}}{2 \lambda}\left[(3 \gamma+2) \rho^{2}-\frac{3 B}{\rho^{\alpha-1}}\right]-\frac{6 \mathcal{U}}{k_{4}^{2} \lambda}, \\
\frac{1}{V} \frac{d}{d t}\left(V H_{i}\right) & =\Lambda-\frac{k_{4}^{2}}{2}\left[(\gamma-1) \rho-\frac{B}{\rho^{\alpha}}\right]-\frac{k_{4}^{2}}{2 \lambda}\left[\gamma \rho^{2}-\frac{B}{\rho^{\alpha-1}}\right]+\frac{2 \mathcal{U}}{k_{4}^{2} \lambda}, i=1.2 .3, \\
\dot{\rho}+3 H(p+\rho) & =0 \\
\dot{\mathcal{U}}+4 H \mathcal{U} & =0
\end{aligned}
$$

Since we have assumed a homogeneous geometry, the effective nonlocal energy density, $\mathcal{P}_{\mu \nu}$, and $\mathcal{Q}_{\mu}$ vanish on the brane, $\mathcal{P}_{\mu \nu}=0$ and $\mathcal{Q}_{\mu}=0$, respectively.

\section{GENERAL SOLUTION OF THE FIELD EQUATIONS FOR THE BIANCHI TYPE I BRANE}

By using the equation of state of the cosmological matter given by Eq. (22), the energy conservation equation Eq. (26) can be integrated exactly, thus giving the time evolution of the energy density of the modified Chaplygin gas filled Bianchi type I brane as

$$
\rho=\left[\frac{B}{1+\gamma}+\frac{C}{V^{(1+\gamma)(1+\alpha)}}\right]^{\frac{1}{1+\alpha}} .
$$

where $C$ is an arbitrary constant of integration.

Integrating Eq. (27) yields

$$
\mathcal{U}=\mathcal{U}_{0} V^{-\frac{4}{3}}
$$

where $\mathcal{U}_{0}$ is an arbitrary constant of integration.

By adding Eqs.(25) we find

$$
\frac{1}{V} \frac{d}{d t}(V H)=\frac{\ddot{V}}{3 V}=\Lambda-\frac{k_{4}^{2}}{2}\left[(\gamma-1) \rho-\frac{B}{\rho^{\alpha}}\right]-\frac{k_{4}^{2}}{2 \lambda}\left[\gamma \rho^{2}-\frac{B}{\rho^{\alpha-1}}\right]+\frac{2 \mathcal{U}}{k_{4}^{2} \lambda},
$$

or, by using the explicit dependence of the energy density on the comoving volume $V$ and defining a new function $\ddot{V}=F(V)$, we can rewrite Eq. (30) in the form

$$
\begin{aligned}
\ddot{V}= & F(V)=3 \Lambda V-\frac{3 k_{4}^{2}}{2} V\left\{(\gamma-1)\left[\frac{B}{1+\gamma}+\frac{C}{V^{(1+\gamma)(1+\alpha)}}\right]^{\frac{1}{1+\alpha}}-B\left[\frac{B}{1+\gamma}+\frac{C}{V^{(1+\gamma)(1+\alpha)}}\right]^{-\frac{\alpha}{1+\alpha}}\right\} \\
& -\frac{3 k_{4}^{2}}{2 \lambda} V\left\{\gamma\left[\frac{B}{1+\gamma}+\frac{C}{V^{(1+\gamma)(1+\alpha)}}\right]^{\frac{2}{1+\alpha}}-B\left[\frac{B}{1+\gamma}+\frac{C}{V^{(1+\gamma)(1+\alpha)}}\right]^{\frac{1-\alpha}{1+\alpha}}\right\}+\frac{6 \mathcal{U}_{0}}{k_{4}^{2} \lambda} V^{-\frac{1}{3}} .
\end{aligned}
$$


The general solution of Eq. (31) is given by

$$
t-t_{0}=\int \frac{d V}{\sqrt{2 \int F(V) d V+C_{1}}}
$$

where $C_{1}$ and $t_{0}$ are arbitrary constant of integration.

Subtracting Eq. (30) from Eqs. (25) we obtain

$$
H_{i}=H+\frac{K_{i}}{V}, i=1,2,3
$$

with $K_{i}, i=1,2,3$ constants of integration satisfying the consistency condition $\sum_{i=1}^{3} K_{i}=0$. From Eq. (33), it is easy to show that

$$
\sum_{i=1}^{3} H_{i}^{2}=3 H^{2}+\frac{K^{2}}{V^{2}}
$$

with $\sum_{i=1}^{3} K_{i}^{2}=K^{2}$.

Therefore for a Bianchi type I induced brane geometry in the presence of the modified Chaplygin gas the general solution of the gravitational field equation can be expressed in the following exact parametric form, with $V \geq 0$ taken as parameter:

$$
\begin{gathered}
\theta=\frac{\sqrt{2 \int F(V) d V+C_{1}}}{V}, \\
a_{i}=a_{i 0} V^{\frac{1}{3}} \exp \left[K_{i} \int \frac{d V}{V \sqrt{2 \int F(V) d V+C_{1}}}\right], i=1,2,3 \\
A=\frac{3 K^{2}}{2 \int F(V) d V+C_{1}}, \\
q=2-\frac{\sigma^{2}=\frac{K^{2}}{2 V^{2}}}{2 \int F(V) d V+C_{1}}
\end{gathered}
$$

where $a_{i 0}, i=1,2,3$, are arbitrary constant of integration. In order for the general solution of the field equations given by Eqs. (35)-(39) be defined for all $V \geq 0$, it is necessary to chose the constants $\alpha$ and $B$, describing the thermodynamic properties of the Chaplygin gas and the integration constant $C$ so that the condition $2 \int F(V) d V+C_{1} \geq 0$ holds for all $V \geq 0$. Taking into account the definition of $V$, given by Eq. (14), and Eqs. ([36), and since the integration constants $K_{i}, i=1,2,3$ satisfy the condition $\sum_{i=1}^{3} K_{i}=0$, it follows that the integration constants $a_{i 0}, i=1,2,3$, have to satisfy the condition $\prod_{i=1}^{3} a_{i 0}=1$.

By introducing two new variables $\tau$ and $v$ by means of the transformations $\tau=\sqrt{3} k_{4} B^{1 / 2(1+\alpha)} t$ and $V=V_{0} v$, with $V_{0}=$ constant, by normalizing the values of the arbitrary integration constants so that $C / B V_{0}^{(1+\gamma)(1+\alpha)}=1$, $4 \mathcal{U}_{0} V_{0}^{-4 / 3} / k_{4}^{4} B^{1 /(1+\alpha)} \lambda=1, C_{1} / 3 V_{0}^{2} k_{4}^{2} B^{1 /(1+\alpha)}=1$, denoting $B^{1 /(1+\alpha)} / \lambda=b, \lambda_{0}=2 \Lambda / 3 k_{4}^{2} B^{1 /(1+\alpha)}$ and

$$
f(v)=\frac{1}{1+\gamma}+\frac{1}{v^{(1+\gamma)(1+\alpha)}}
$$

it follows that Eq. (32) can be written in the form

$$
\tau-\tau_{0}=\int \frac{d v}{\sqrt{\int\left\{\lambda_{0} v-v\left[(\gamma-1) f^{\frac{1}{1+\alpha}}-f^{-\frac{\alpha}{1+\alpha}}\right]-b v\left[\gamma f^{\frac{2}{1+\alpha}}-f^{\frac{1-\alpha}{1+\alpha}}\right]+v^{-\frac{1}{3}}\right\} d v+1}}
$$


where $\tau_{0}=\sqrt{3} k_{4} B^{1 / 2(1+\alpha)} t_{0}$.

With the use of Eqs. (28), (33) and (35), from Eq. (24) it follows that the arbitrary integration constants $K_{i}$, $i=1,2,3$ and $C_{1}$ must satisfy the consistency condition

$$
K^{2}=\frac{2 C_{1}}{3},
$$

Scalar fields are supposed to play a fundamental role in the evolution of the early universe. The Chaplygin gas model can be also described from a field theoretical point of view by introducing a scalar field $\phi$ and a self interacting potential $U(\phi)$, with the Lagrangian [6], 7], 10], 13], 37]

$$
L_{\phi}=\frac{1}{2} \dot{\phi}^{2}-U(\phi) .
$$

The energy density and the pressure associated to the scalar field $\phi$ are given by

$$
\rho_{\phi}=\frac{\dot{\phi}^{2}}{2}+U(\phi)=\rho,
$$

and

$$
p_{\phi}=\frac{\dot{\phi}^{2}}{2}-U(\phi)=\gamma \rho-\frac{B}{\rho^{\alpha}},
$$

respectively.

In view of Eqs.(44), (45) and (28) we have

$$
\dot{\phi}^{2}=(1+\gamma)\left[\frac{B}{1+\gamma}+\frac{C}{V^{(1+\gamma)(1+\alpha)}}\right]^{\frac{1}{1+\alpha}}-B\left[\frac{B}{1+\gamma}+\frac{C}{V^{(1+\gamma)(1+\alpha)}}\right]^{-\frac{\alpha}{1+\alpha}},
$$

and

$$
U(\phi)=\frac{(1-\gamma)}{2}\left[\frac{B}{1+\gamma}+\frac{C}{V^{(1+\gamma)(1+\alpha)}}\right]^{\frac{1}{1+\alpha}}+\frac{B}{2}\left[\frac{B}{1+\gamma}+\frac{C}{V^{(1+\gamma)(1+\alpha)}}\right]^{-\frac{\alpha}{1+\alpha}}
$$

respectively.

By integrating Eq. (46), we obtain

$$
\phi-\phi_{0}=\int \sqrt{\frac{(1+\gamma)\left[\frac{B}{1+\gamma}+\frac{C}{V^{(1+\gamma)(1+\alpha)}}\right]^{\frac{1}{1+\alpha}}-B\left[\frac{B}{1+\gamma}+\frac{C}{V^{(1+\gamma)(1+\alpha)}}\right]^{-\frac{\alpha}{1+\alpha}}}{2 \int F(V) d V+C_{1}}} d V
$$

where $\phi_{0}$ is an arbitrary constant of integration.

\section{DISCUSSIONS AND FINAL REMARKS}

In the present paper we have considered Chaplygin gas filled Bianchi type I brane worlds in the framework of the Randall-Sundrum scenario 24]. The Randall-Sundrum mechanism was originally motivated as a possible mechanism for evading Kaluza-Klein compactification by localizing gravity in the presence of an uncompactified extra dimension. In this model, the 5-dimensional bulk space-time is assumed to be vacuum except for the presence of a cosmological constant. Matter fields on the brane are regarded as responsible for the dynamics of the brane. The quadratic terms in the energy-momentum tensor, due to the form of the Gauss-Codazzi equations, may be important in the very early stages of the Universe, leading to major changes in the dynamics of the Universe. The general solution of the gravitational field equations on the brane can be obtained for both discussed brane geometries in an exact parametric form, with the comoving volume element $V$ taken as parameter.

By obtaining the general solution of the field equations we have assumed the presence of a non-zero effective cosmological constant $\Lambda$ on the brane. However, the most attractive feature of the Chaplygin gas is that it could explain the main observational properties of the Universe without appealing to an effective cosmological constant. To 
see if this is indeed the case in the anisotropic brane world models, in the following we will assume that on the brane $\Lambda=0$. This constraint imposes the following relation between the vacuum energies in the bulk and on the brane:

$$
\Lambda_{5}=-\frac{k_{5}^{2}}{6} \lambda^{2}
$$

Therefore for $\lambda_{0}=0$ and for a fixed $\gamma$ and $\alpha$, the dynamics of the anisotropic brane Universes is controlled by the parameter $b=B^{1 /(1+\alpha)} / \lambda$, the ratio of the Chaplygin gas equation of state parameter and the vacuum energy on the brane.

From the equation of state of the Chaplygin gas with $\gamma=0$ it follows that for the critical values $p_{c}$ and $\rho_{c}$ of the pressure and density the parameter $w_{c}=p_{c} / \rho_{c}$ is given by $w_{c}=-B / \rho_{c}^{\alpha+1}$. Evaluating this relation at the present time gives $B=-w_{c 0} \rho_{c 0}^{\alpha+1}$. The Chaplygin gas behaves like a cosmological constant for $w_{c 0}=-1$, which fixes the constant $B$ as $B=\rho_{c 0}^{\alpha+1}=\left(3 H_{0}^{2} / 8 \pi G\right)^{\alpha+1}$, where $H_{0}=3.24 \times 10^{-18} h \mathrm{~s}^{-1}, 0.5 \leq h \leq 1$, is the Hubble constant [3], [4]. The definition of the dimensionless time parameter $\tau=\sqrt{3} k_{4} B^{1 / 2(\alpha+1)} t$ then gives $\tau=3 H_{0} t$, or $t \approx 10^{17} h^{-1} \times \tau$ $\mathrm{s}^{-1}$.

Generally, the obtained solution of the gravitational field equations describes an expanding brane Universe. The variation of the expansion scalar $\theta$ as a function of the dimensionless time parameter $\tau$ is represented in Fig. 1.

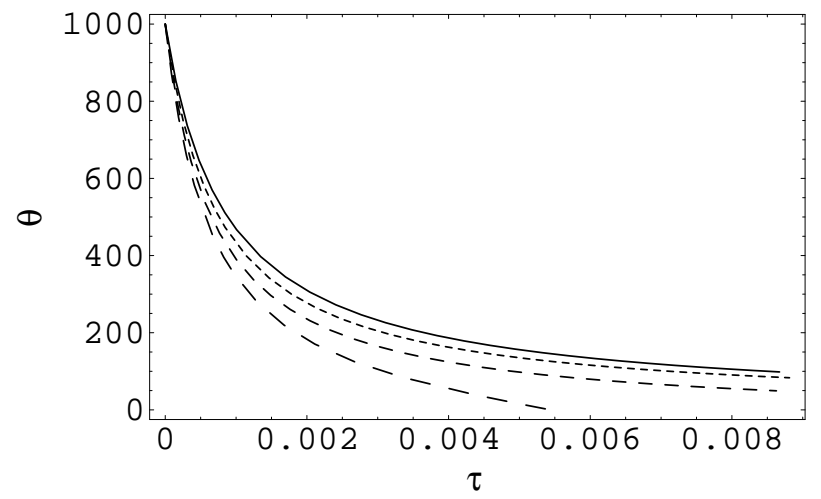

FIG. 1: Expansion scalar $\theta$ of the Chaplygin gas filled Bianchi type I brane universe as a function of the dimensionless time parameter $\tau$ for $\gamma=1 / 3, \alpha=0.5$ and $b=0.01$ (solid curve), $b=0.02$ (dotted curve), $b=0.03$ (dashed curve) and $b=0.04$ (long dashed curve). We have assumed a vanishing effective cosmological constant on the brane, $\lambda_{0}=0$. The relation between $\tau$ and the cosmic time $t$ is $t \approx 10^{17} h^{-1} \times \tau \mathrm{s}^{-1}, 0.5 \leq h \leq 1$.

For a Chaplygin type cosmological fluid confined on the brane, the early cosmological evolution in anisotropic Bianchi type I geometry is fundamentally changed by the inclusion of the terms proportional to the square of the energy density and energy density-pressure products. The time variation of the mean anisotropy parameter of the Bianchi type I space-time is represented, for $\gamma=1 / 3, \alpha=0.5$ and for different values of the parameter $b$, in Fig. 2. At high densities the brane Universe starts its evolution from an isotropic state, with $A\left(t_{0}\right)=0$. The anisotropy increases and reaches a maximum value after a finite time interval $t_{\max }$, and for $t>t_{\max }$, the mean anisotropy is a monotonically decreasing function, tending to zero in the large time limit. This behavior is in sharp contrast to the usual general relativistic evolution (in this case the quadratic contribution to the energy-momentum tensor vanishes), in which the Universe is born in a state of maximum anisotropy.

The time variation of the shear parameter $\sigma^{2}$ is represented, as a function of the dimensionless time $\tau$ and for different values of $b$, in Fig. 3 .

The shear is a monotonically decreasing function of the cosmological time, and in the limit of large $t, t \rightarrow \infty$, corresponding to the isotropic limit, $\sigma^{2}$ tends to zero. In the limit of small times, since $\sigma^{2} \propto V^{-2}$, the shear has a singular behavior, tending to infinity, $\lim _{t \rightarrow 0} \sigma^{2} \rightarrow \infty$.

In the initial stage the evolution of the Chaplygin gas filled Bianchi type I brane universe is non-inflationary, but in the large time limit the brane Universe ends in an accelerating stage. In Fig. 4 we present the dynamics of the deceleration parameter for different values of $b$ and for $\gamma=1 / 3$ and $\alpha=0.5$. 


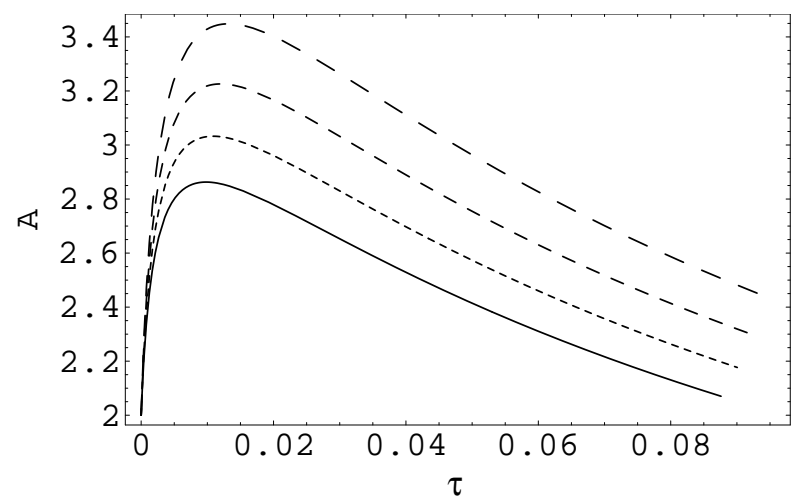

FIG. 2: Anisotropy parameter $A$ of the Chaplygin gas filled Bianchi type I brane universe as a function of the dimensionless time parameter $\tau$ for $\gamma=1 / 3, \alpha=0.5$ and $b=0.01$ (solid curve), $b=0.011$ (dotted curve), $b=0.012$ (dashed curve) and $b=0.013$ (long dashed curve). We have assumed a vanishing effective cosmological constant on the brane, $\lambda_{0}=0$. The relation between $\tau$ and the cosmic time $t$ is $t \approx 10^{17} h^{-1} \times \tau \mathrm{s}^{-1}, 0.5 \leq h \leq 1$.

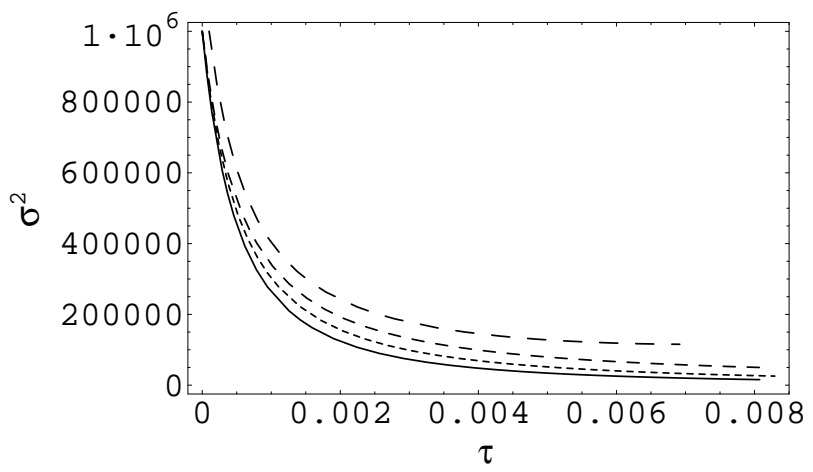

FIG. 3: Shear scalar $\sigma^{2}$ of the Chaplygin gas filled Bianchi type I brane universe as a function of the dimensionless time parameter $\tau$ for $\gamma=1 / 3, \alpha=0.5$ and $b=0.01$ (solid curve), $b=0.02$ (dotted curve), $b=0.03$ (dashed curve) and $b=0.04$ (long dashed curve). We have assumed a vanishing effective cosmological constant on the brane, $\lambda_{0}=0$. The relation between $\tau$ and the cosmic time $t$ is $t \approx 10^{17} h^{-1} \times \tau \mathrm{s}^{-1}, 0.5 \leq h \leq 1$.

The time variation of the scalar field associated with the Chaplygin gas is represented for fixed $\alpha$ and $\gamma$ and different values of the parameter $b$ in Fig. 5. The scalar field is a monotonically decreasing function of time.

The dependence of the potential $U(\phi)$ on the scalar field $\phi$ is represented in Fig. 6.

In the limit of high densities, the equation of state of the Chaplygin gas reduces to the linear barotropic equation of state of the relativistic matter, $p=\gamma \rho$. In this case the density dependence on the comoving volume is given by $\rho=\rho_{0} / V^{\gamma+1}, \rho_{0}=$ constant, and the equation describing the time variation of the comoving volume reduces to

$$
t-t_{0}=\int \frac{d V}{\sqrt{3 k_{4}^{2} \rho_{0} V^{1-\gamma}+\frac{3 k_{4}^{2}}{2 \lambda} \rho_{0}^{2} V^{-2 \gamma}+\frac{18 \mathcal{U}_{0}}{k_{4}^{2} \lambda} V^{2 / 3}+C_{1}}} .
$$

In the limit of very high matter densities, which can be described by the strong equation of state for the hot nucleonic gas, it is assumed that $p=\rho$. It is believed that matter actually behaves in this manner at densities above about ten times the nuclear density, that is, at densities greater than $10^{17} \mathrm{~g} / \mathrm{cm}^{3}$ and at temperatures $T=\left(\rho c^{2} / \sigma_{\text {rad }}\right)^{1 / 4}>10^{13}$ $\mathrm{K}$, where $\sigma_{\text {rad }}$ is the radiation constant [38]. Therefore in this case $\gamma=1$. In the limit of small $V$, the term $V^{-2}$ will dominate in the integral in Eq. (50). By maintaining only this term, the integration can be done easily and the time dependence of the relevant geometrical and physical parameters can be obtained as

$$
V=\left(\frac{6 k_{4}^{2} \rho_{0}^{2}}{\lambda}\right)^{1 / 4} t^{1 / 2}
$$




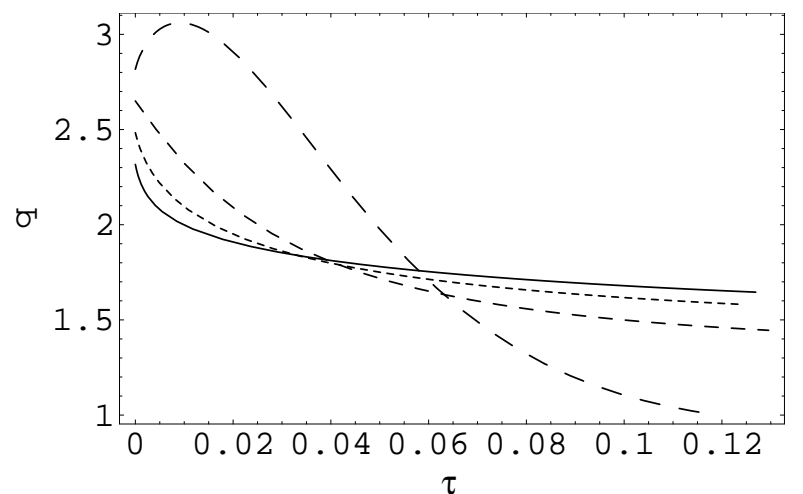

FIG. 4: Deceleration parameter $q$ of the Chaplygin gas filled Bianchi type I brane universe as a function of the dimensionless time parameter $\tau$ for $\gamma=1 / 3, \alpha=0.5$ and $b=0.01$ (solid curve), $b=0.015$ (dotted curve), $b=0.02$ (dashed curve) and $b=0.025$ (long dashed curve). We have assumed a vanishing effective cosmological constant on the brane, $\lambda_{0}=0$. The relation between $\tau$ and the cosmic time $t$ is $t \approx 10^{17} h^{-1} \times \tau \mathrm{s}^{-1}, 0.5 \leq h \leq 1$.

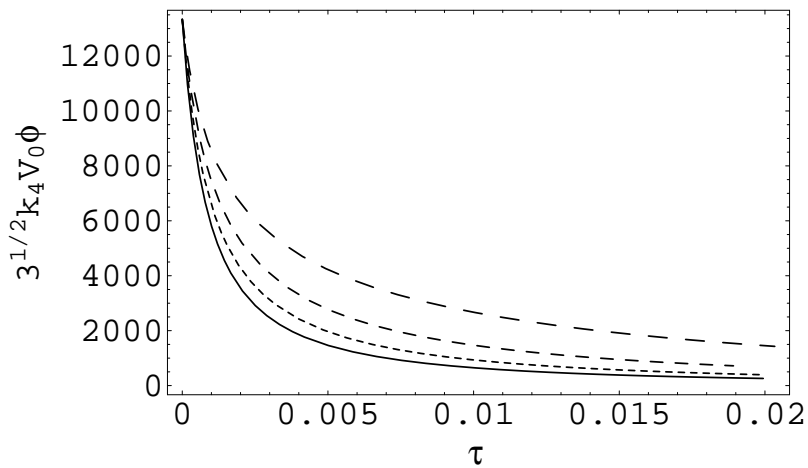

FIG. 5: Chaplygin gas associated scalar field $\phi$ evolution on the Bianchi type I brane universe as a function of the dimensionless time parameter $\tau$ for $\gamma=1 / 3, \alpha=0.5$ and $b=0.005$ (solid curve), $b=0.01$ (dotted curve), $b=0.015$ (dashed curve) and $b=0.02$ (long dashed curve). We have assumed a vanishing effective cosmological constant on the brane, $\lambda_{0}=0$. The relation between $\tau$ and the cosmic time $t$ is $t \approx 10^{17} h^{-1} \times \tau \mathrm{s}^{-1}, 0.5 \leq h \leq 1$.

$$
\begin{gathered}
\theta=\frac{1}{2 t}, \\
a_{i}=a_{i 0} t^{1 / 6} \exp \left[2 K_{i}\left(\frac{6 k_{4}^{2} \rho_{0}^{2}}{\lambda}\right)^{-1 / 4} t^{1 / 2}\right], i=1,2,3, \\
A=\frac{12 K^{2}}{\left(\frac{6 k_{4}^{2} \rho_{0}^{2}}{\lambda}\right)^{1 / 2}} t \\
\sigma^{2}=\frac{K^{2}}{2\left(\frac{6 k_{4}^{2} \rho_{0}^{2}}{\lambda}\right)^{1 / 2} \frac{1}{t},} \\
q=5,
\end{gathered}
$$

where we have assumed the initial conditions $t_{0}=0$ and $V(0)=0$.

In the opposite limit of small densities, the equation of state of the Chaplygin gas becomes $p=-B / \rho^{\alpha}$, corresponding to the limit $\gamma \rightarrow 0$. In this case the equation describing the time dependence of the comoving volume $V$ is 


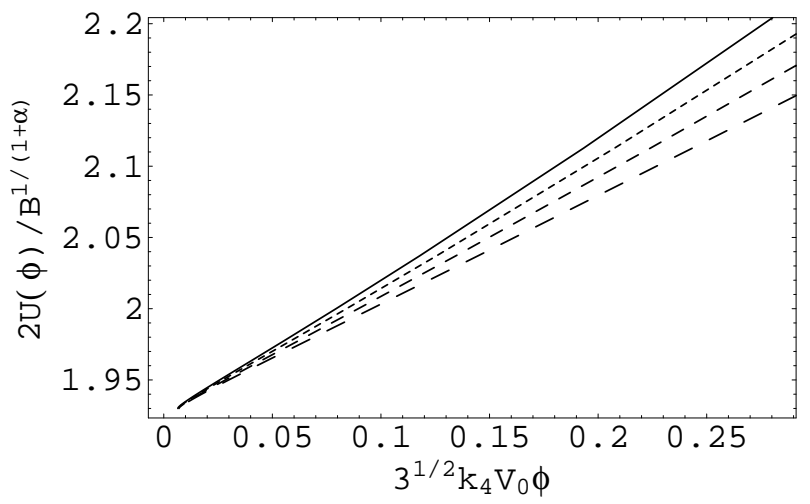

FIG. 6: The potential $U(\phi)$ of the Chaplygin gas associated scalar field as a function of the scalar field $\phi$ for $\gamma=1 / 3, \alpha=0.5$ and $b=0.005$ (solid curve), $b=0.01$ (dotted curve), $b=0.015$ (dashed curve) and $b=0.02$ (long dashed curve). We have assumed a vanishing effective cosmological constant on the brane, $\lambda_{0}=0$.

given by

$$
\ddot{V}=\frac{3 k_{4}^{2}}{2} V\left\{\left[B+\frac{C}{V^{1+\alpha}}\right]^{\frac{1}{1+\alpha}}+B\left[B+\frac{C}{V^{1+\alpha}}\right]^{-\frac{\alpha}{1+\alpha}}\right\}+\frac{3 k_{4}^{2} B}{2 \lambda} V\left[B+\frac{C}{V^{1+\alpha}}\right]^{\frac{1-\alpha}{1+\alpha}}+\frac{6 \mathcal{U}_{0}}{k_{4}^{2} \lambda} V^{-\frac{1}{3}}
$$

In the limit of large $V$ the condition $B>>C / V^{1+\alpha}$ is always fulfilled, and the general solution of Eq. (57) can be expressed as

$$
t-t_{0}=\int \frac{d V}{\sqrt{\Lambda_{c}^{2} V^{2}+\frac{18 \mathcal{U}_{0}}{k_{4}^{2} \lambda} V^{2 / 3}+C_{1}}}
$$

where we have denoted

$$
\Lambda_{c}=\sqrt{3 k_{4}^{2} B^{1 /(1+\alpha)}\left(1+\frac{B^{1 /(1+\alpha)}}{2 \lambda}\right)} .
$$

In the same limit $V \rightarrow \infty$ we also have $\Lambda_{c} V^{2}>>18 \mathcal{U}_{0} V^{2 / 3} / k_{4}^{2} \lambda+C_{1}$ and therefore Eq. (58) can be immediately integrated to give

$$
V=V_{1} \exp \left[\Lambda_{c}\left(t-t_{0}\right)\right] .
$$

where $V_{1}$ is an arbitrary constant of integration.

The time evolution of the physical and geometrical quantities in the limit of the large comoving volume is given by

$$
\begin{gathered}
\theta=\Lambda_{c}, \\
a_{i}=a_{i 0} \exp \left[\frac{\Lambda_{c}}{3}\left(t-t_{0}\right)\right] \exp \left\{-\frac{K_{i}}{\Lambda_{c} V_{1}} \exp \left[-\Lambda_{c}\left(t-t_{0}\right)\right]\right\}, i=1,2,3, \\
A=\frac{3 K^{2}}{\Lambda_{c}^{2} V_{1}^{2}} \exp \left[-2 \Lambda_{c}\left(t-t_{0}\right)\right] \\
\sigma^{2}=\frac{K^{2}}{2 V_{1}^{2}} \exp \left[-2 \Lambda_{c}\left(t-t_{0}\right)\right], \\
q=-1 .
\end{gathered}
$$


In the limit of large times the mean anisotropy parameter tends to zero, $A \rightarrow 0$, and the Chaplygin gas filled Bianchi type I Universe ends in an isotropic state, corresponding to a de Sitter type Universe. This shows that the Chaplygin gas model offers a real possibility for replacing the effective cosmological constant on the brane.

[1] S. Perlmutter et al., Astrophys. J. 517, 565 (1998); A. G. Riess et al., Astron. J. 116, 109 (1998).

[2] P. de Bernardis et al., Nature 404, 995 (2000); S. Hanany et al., Astrophys. J. 545, L5 (2000).

[3] P. J. E. Peebles and B. Ratra, Rev. Mod. Phys. 75, 559 (2003).

[4] T. Padmanabhan, Phys. Repts. 380, 235 (2003).

[5] T. Matos and A. Urena-Lopez, Class. Quantum Grav. 17, L75 (2000); C. Wetterich, Phys. Rev. D. 65, 123512; T. Padmanabhan and T. R. Choudhury, Phys. Rev. D 66, 081301 (2002).

[6] A. Kamenshchik, U. Moschella and V. Pasquier, Phys. Lett. B 511, 265 (2001).

[7] M. C. Bento, O. Bertolami and A. Sen, Phys. Rev. D 66, 043507 (2002).

[8] A. Kamenshchik, U. Moschella and V. Pasquier, Phys. Lett. B 487, 7 (2000).

[9] S. K. Kama, Phys. Lett. B 424, 39 (1998).

[10] N. Bilic, G. B. Tupper and R. D. Viollier, Phys. Lett. B 535, 17 (2002).

[11] M. Novello, M. Makler, L. S. Werneck and C. A. Romero, Phys. Rev. D 71, 043515 (2005).

[12] D. Carturan and F. Finelli, Phys. Rev. D 68, 103501 (2003); R. Bean and O. Dore, Phys. Rev. D 68, 023515 (2003); L. M. G. Beca, P. P. Avelino, J. P. M. de Carvalho and C. J. A. P. Martins, Phys. Rev. D 67, 101301 (2003); M. C. Bento, O. Bertolami and A. A. Sen, Phys. Rev. D 70 083519, (2004); R. R. R. Reis, I. Waga, M. O. Calvao and S. E. Joras, Phys. Rev. D 68061302 (2003); P. P. Avelino, L. M. G. Beca, J. P. M. de Carvalho and C. J. A. P. Martins, JCAP 0309002 (2003); G. M. Kremer, Phys. Rev. D 68123507 (2003); T. Multamaki, M. Manera and E. Gaztanaga, Phys. Rev. D 69, 023004 (2004); M. Szydlowski and W. Czaja, Phys. Rev. D 69023506 (2004); P. P. Avelino, L. M. G. Beca, J. P. M. de Carvalho, C. J. A. P. Martins and E.J. Copeland, Phys. Rev. D 69041301 (2004).

[13] O. Bertolami, A. A. Sen, S. Sen and P. T. Silva, Mon. Not. Roy. Astron. Soc. 353, 329 (2004)

[14] M. Makler, S. Quinet de Oliveira and I. Waga, Phys. Lett. B 555, 1 (2003); J. V. Cunha, J. S. Alcaniz, J. A. S. Lima, Phys. Rev. D 69, 083501 (2004); M. C. Bento, O. Bertolami, N. M. C. Santos and A. A. Sen, Phys. Rev. D 71, 063501 (2005).

[15] M. C. Bento, O. Bertolami and A. A. Sen, Phys. Lett. B 575, 172 (2003); M. C. Bento, O. Bertolami and A. A. Sen, Phys. Rev. D 67, 063003 (2003); L. Amendola, F. Finelli, C. Burigana, D. Carturan, JCAP 0307, 005 (2003).

[16] A. Dev, D. Jain and J. S. Alcaniz, Phys. Rev. D 67, 023515 (2003); A. Dev, D. Jain and J. S. Alcaniz, Astron. Astrophys. 417, 847 (2004); P. T. Silva and O. Bertolami, Astrophys. J. 599, 829 (2003).

[17] J. S. Alcaniz, D. Jain and A. Dev, Phys. Rev. D 67, 043514 (2003).

[18] J. V. Cunha, J. A. S. Lima and J. S. Alcaniz, Phys. Rev. D 69, 083501 (2004); Z.-H. Zhu, Astron. Astrophys. 423, 421 (2004).

[19] L. P. Eisenhart, Riemannian geometry, Princeton, Princeton University Press (1949).

[20] K. Akama, Pregeometry, in Lect. Notes Phys. 176, 267 (1983).

[21] V. A. Rubakov and M. E. Shaposhnikov, Phys. Lett. B125, 136 (1983).

[22] I. Bandos and W. Kummer, Int. J. Mod. Phys. A 14, 4881 (1999).

[23] M. Pavsic and V. Tapia, gr-qc/0010045 (2000).

[24] L. Randall and R. Sundrum, Phys. Rev. Lett. 83, 3370 (1999); L. Randall and R. Sundrum, Phys. Rev. Lett 83, 4690 (1999).

[25] R. Maartens, Living Rev. Rel. 7, 7 (2004).

[26] T. Shiromizu, K. Maeda and M. Sasaki, Phys. Rev. D62, 024012 (2000); M. Sasaki, T. Shiromizu and K. Maeda, Phys. Rev. D62, 024008 (2000).

[27] K. Maeda and D. Wands, Phys. Rev. D 62, 124009 (2000); R. Maartens, Phys. Rev. D 62, 084023 (2000); A. Campos and C. F. Sopuerta, Phys. Rev. D64, 104011 (2001); D. Langlois, Phys. Rev. Lett. 86, 2212 (2001); C.-M. Chen, T. Harko and M. K. Mak, Phys. Rev. D 64, 124017 (2001); L. Anchordoqui, J. Edelstein, C. Nunez, S. P. Bergliaffa, M. Schvellinger, M. Trobo and F. Zyserman, Phys. Rev. D 64, 084027 (2001); J. D. Barrow and R. Maartens, Phys. Lett. B 532, 153 (2002); H. Kudoh and T. Tanaka, Phys. Rev. D 65, 104034 (2002); H. A. Bridgman, K. A. Malik and D. Wands, Phys. Rev. D 65, 043502 (2002); C.-M. Chen, T. Harko, W. F. Kao and M. K. Mak, Nucl. Phys. B 636, 159 (2002); M. Szydlowski, M. P. Dabrowski and A. Krawiec, Phys. Rev. D 66, 064003 (2002); T. Harko and M. K. Mak , Class. Quantum Grav. 20, 407 (2003), C.-M. Chen, T. Harko, W. F. Kao and M. K. Mak, JCAP 0311, 005 (2003); Iver Brevik, Kazuo Ghoroku, Masanobu Yahiro, Phys. Rev. D 70 064012, (2004).

[28] N. Dadhich and S. G. Ghosh, Phys. Lett. B518, 1 (2001); M. G. Santos, F. Vernizzi and P. G. Ferreira, Phys. Rev. D 64, 063506 (2001); M. Bruni, C. Germani and R. Maartens, Phys. Rev. Lett. 87, 231302 (2001); H.-C. Kim, S.-H. Moon and J. H. Yee, JHEP 0202, 046 (2002); M Govender and N. Dadhich, Phys. Lett. B 538, 233 (2002); T. Wiseman, Class. Quant. Grav. 19, 3083 (2002); R. Neves and C. Vaz, Phys. Rev. D 66, 124002 (2002); L. A. Anchordoqui, H. Goldberg and A. D. Shapere, Phys. Rev. D 66, 024033 (2002); H. Kudoh, T. Tanaka and T. Nakamura, Phys. Rev. D 68, 024035 (2003); T. Harko and M. K. Mak, Phys. Rev. D 69, 064020 (2004).

[29] M. K. Mak and T. Harko, Phys. Rev. D 70, 024010 (2004). 
[30] R. Maartens, V. Sahni and T. D. Saini,Phys. Rev. D 63, 063509 (2001); A. V. Toporensky, Class. Quant. Grav. 18, 2311 (2001); A. V. Frolov, Phys. Lett. B 514, 213 (2001); M. G. Santos, F. Vernizzi and P. G. Ferreira, Phys. Rev. D 64, 063506 (2001); T. Harko and M. K. Mak, Class. Quant. Grav. 20, 407 (2003).

[31] C.-M. Chen, T. Harko and M. K. Mak, Phys. Rev. D 64, 044013 (2001).

[32] A. Campos and C. F. Sopuerta, Phys. Rev. D 63, 104012.

[33] A. Coley, Phys. Rev. D 66023512 (2002).

[34] A. Coley, Class. Quantum Grav. 19, L45 (2002).

[35] T. Harko and M. K. Mak, Class. Quantum. Grav. 21, 1489 (2004).

[36] K. Maeda and D. Wands, Phys. Rev. D 62, 124009 (2000).

[37] U. Debnath, A. Banerjee and S. Chakraborty, Class. Quant. Grav. 21, 5609 (2004).

[38] Ya. B. Zeldovich and I. D. Novikov, Relativistic Astrophysics, Univ. of Chicago Press, Chicago, Ill (1971). 